\title{
La política de población en el Perú: Breve historia reciente.
}

Population policy in Peru: Brief recent history.

\author{
Mario Gustavo Berrios Espezúa
}

Universidad Nacional de San Agustín. Arequipa Perú.

\section{INFORMACIÓN}

\section{Historia del Artículo \\ Recepción: 07/02/2020 \\ Revisión: 05/05/2020}

Aceptación: 20/05/2020

\section{Palabras Clave}

Demografía, gestión pública, Política de población, política pública

\section{Key Words}

Demography, public management, population policy, public politics.

\section{DOI}

https://doi.org/10.35286/veritas. v21i 2.270

\section{RESUMEN}

El proceso de elaboración de la política de población es un compromiso asumido por el Estado peruano a través de acuerdos y tratados internacionales de los cuales forma parte. En base a éstos, el Estado, para aplicar una apropiada política pública de población debe planificar y ejecutar acciones públicas en lo referente a la dinámica, estructura, volumen y distribución de la población sobre el territorio nacional. En ese sentido, dicha política se da dentro de un marco más amplio en favor del desarrollo socioeconómico y no de forma aislada. En el presente estudio reflexionamos acerca de los antecedentes de la Política de Población peruana, la actual Ley de Política de Población y los principales elementos del Plan Nacional de Población 2010-2014 (el mismo que actualmente está en vigencia). Para poder cumplir con estos objetivos analizamos los documentos oficiales del Estado Peruano en materia de población. En síntesis, los principales hallazgos del presente estudio nos muestran la preocupación del Estado peruano por elaborar un marco normativo en materia de política de población; sin embargo, la principal dificultad se observa en el cumplimiento e implementación de dicho marco normativo.

\begin{abstract}
The process of preparing the population policy is a commitment assumed by the Peruvian State through international agreements and treaties of which it is a part. Based on these, the State, in order to apply an appropriate public population policy, must plan and execute public actions regarding the dynamics, structure, volume and distribution of the population over the national territory. In that sense, this policy occurs within a broader framework in favor of socio-economic development and not in isolation. In this study we reflect on the background of the Peruvian Population Policy, the current population policy law and the main elements of the National Population Plan 2010-2014 (the same one that is currently in force). In order to meet these objectives, we analyze the official documents of the Peruvian State regarding population. In summary, the main findings of this study show us the concern of the Peruvian State to develop a regulatory framework in terms of population policy; However, the main difficulty is observed in the compliance and implementation of said regulatory framework.
\end{abstract}

\section{Antecedentes de la ley de política nacional de población}

El 31 de agosto de 1976, el gobierno Revolucionario de las Fuerzas Armadas, presidido por el General Francisco Morales Bermúdez define y adopta una serie de lineamientos de política de población. En referidos lineamientos se define a la política de población como:

[...]el conjunto de objetivos y acciones que tienen una incidencia directa sobre el volumen, crecimiento, estructura y distribución de la población en el territorio nacional, respetando los derechos de las personas y con la finalidad de contribuir a que la población en su conjunto logre alcanzar sus aspiraciones de un auténtico desarrollo y seguridad integral (Ministerio de Salud, 1976: 49).
Los principales objetivos que se formularon en el Decreto Supremo 00625-76-MS del 31 de agosto de 1976, fueron: lograr un crecimiento poblacional que esté en armonía con la libre decisión de la población sobre la dimensión familiar y que contribuya a hacer efectivos los esfuerzos que la sociedad peruana realiza para alcanzar los niveles de desarrollo humano que aspira. En segundo lugar, lograr una reducción significativa de la morbi-mortalidad, especialmente de la madre y del niño, que permita elevar la calidad y esperanza de vida de toda la población. Y, en tercer lugar, lograr una mejor distribición de la población en el territorio, en concordancia con los objetivos de desarrollo regional y de la seguridad nacional.

Para poder cumplir con mencionados objetivos, se propusieron los siguientes lineamientos de programación y evaluación: 
- Adecuar la estructura económica y social del país al servicio de toda la población.

- Propender al pleno empleo como expresión de promoción humana y de justicia social.

- Orientar el desarrollo urbano y replantear el problema de la vivienda y acondicionamiento habitacional.

- Promover la organización y desarrollo del espacio económico.

- Apoyar el desarrollo de la conciencia familiar, social y nacional, y promover el ejercicio de la participación plena en el desarrollo del país.

Es importante destacar el hecho de que la política de población fue considerada como una política de carácter multisectorial; es decir, que su implementación y cumplimiento debería corresponder a varios ministerios dentro del Poder Ejecutivo.

Años después, durante el segundo gobierno del arquitecto Fernando Belaúnde Terry (1980-1985), se aprobaron un conjunto de normas acerca de la planificación familiar y el 20 de noviembre de 1980 , por D. S. N $049-80-\mathrm{PCM}$, se creó el Consejo Nacional de Población, el mismo que dependía de la presidencia del Consejo de Ministros. La creación del Consejo Nacional de Población fue un avance muy importante en materia de institucionalización de la política de población, más aún porque contó con un grupo selecto de intelectuales y especialistas en la materia. ${ }^{2}$ Las principales acciones que este Consejo realizó estuvieron orientadas a la elaboración de libros, folletos, materiales educativos, organización de eventos académicos, etc., los mismos que incentivaron el debate en torno al tema poblacional. Según reflexiones de diversos autores como Marcos Cueto (2006), José Donayre, Roger Guerra y Luis Sobrevilla (2012) y Raúl Necochea (2016), consideran que este proceso llevó a que el Perú fuera considerado un país modelo (junto con México) en cuanto a políticas de población se refiere.

\section{La ley de política nacional de población}

A principios de 1985, el Consejo Nacional de Población inicia una serie de acciones para debatir y reformular los lineamientos de la política de población elaborados en 1976 y por lo tanto darle mayor institucionalidad legal al tema, con la elaboración de una ley específica.

El debate de esta propuesta de ley, es descrito por José Donayre, Roger Guerra y Luis Sobrevilla (2012) de la siguiente manera:

[...] el Presidente Belaúnde solicita al Congreso de la República la formación de una comisión para la elaboración de un proyecto de ley de política de población. [...], el Congreso conforma una comisión multipartidaria a fin de elaborar un anteproyecto de ley que cuente con amplio consenso en el tema [...] En el Consejo Nacional de Población, designado el Secretariado Técnico, se forma una comisión integrada por sus expertos y se convoca como asesor al sacerdote, demógrafo y economista Juan Julio Wicht. La comisión parlamentaria revisa y debate el anteproyecto en varias sesiones, que se realizaron en la sede del Congreso, y consulta también el anteproyecto con representantes de la Iglesia Católica y los Institutos Armados. Cuando el anteproyecto estaba concluido, y antes de su promulgación como ley, los sectores conservadores y religiosos, igual que ocurrió con las Normas de Planificación Familiar, hicieron una serie de denuncias, que en este caso llegaron a la televisión y a los medios impresos. Esto generó el primer gran debate sobre estos temas en la prensa nacional (Donayre, Guerra y Sobrevilla, 2012: 167).

Finalmente, el 6 de julio de 1985, en las postrimerías del gobierno de Fernando Belaúnde Terry, se promulgó la Ley de Política Nacional de Población, mediante Decreto Legislativo $\mathrm{N}^{\circ}$ 346. En el título preliminar de dicha ley se menciona que "La política Nacional de Población tiene por objeto planificar y ejecutar las acciones del Estado relativas al volumen, estructura, dinámica y distribución de la población en el territorio nacional".

De la misma forma, en el Título I se establecen los objetivos de la ley:

- Promover una equilibrada y armónica relación entre el crecimiento, la estructura y la distribución de la población, y el desarrollo económico y social, teniendo en cuenta que la economía está al servicio del hombre.

- Promover y asegurar la decisión libre, informada y responsable de las personas y las parejas sobre el número y espaciamiento de los nacimientos, proporcionando para ello los servicios educativos y de salud, para contribuir con la estabilidad y solidaridad de la familia y mejorar la calidad de la vida.

- Lograr una reducción significativa de la morbilidad y mortalidad, especialmente entre las madres y los niños, mejorando los niveles de salud y de vida de la población. - Lograr una mejor distribución de la población en el territorio en concordancia con el uso adecuado de los recursos, el desarrollo regional y la seguridad nacional.

En el Título II se desarrollan las 5 políticas de población. La primera de ellas está referida a la familia, dicha política está orientada a garantizar el ejercicio de la paternidad responsable, garantizar la seguridad y bienestar de los niños y personas de la tercera edad, así como destinar acciones al desarrollo integral de la mujer, la madre trabajadora y revalorar el trabajo doméstico. La segunda política es la educación en población, la misma que debe desarrollarse en coordinación con el Ministerio de Educación, para garantizar el impulso de programas de educación en materia poblacional, en todos los niveles del sistema educativo, los mismos que deben ser permanentes y activos; la educación en materia poblacional considera la educación familiar, sexual y la educación sobre el medio ambiente. La tercera política hace referencia a la comunicación social, en esta política el Estado se compromete a promover la difusión de los programas poblacionales a través de los diversos medios de comunicación, los mismos que deben contribuir a la labor educativa. La cuarta política, denominada salud y población,

${ }^{1}$ Correspondencia: mberriose@unsa.edu.pe

${ }^{2}$ Se destacan el sociólogo Mario Torres, al antropólogo Carlos Aramburú, la demógrafa Sandra Vallenas y el especialista en asuntos de género Marfil Francke, entre otros 
tiene una fuerte interrelación con el Ministerio de Salud, para garantizar la paternidad responsable y la planificación familiar en todos los establecimientos del sector salud. Del mismo modo adoptar medidas para disminuir la morbi-mortalidad, la mortalidad infantil y promover el mejoramiento del estado nutricional de la población, el cuidado de la madre gestante y la lactancia materna. Finalmente, la quinta política, desarrollo nacional y distribución de la población, marcó un hecho importante, ya que por primera vez se habla, desde el Estado, de medidas para planificar el desarrollo urbano y la distribución adecuada de la población, lo que hoy llamamos ordenamiento territorial.

En el Título III se recalca el hecho de que la política de población tiene un carácter multisectorial y que es el Consejo Nacional de Población la institución estatal encargada de la elaboración de los planes y programas de población, en coordinación con los organismos del Estado que competa, los mismos que tienen la obligación de brindar información y asistencia técnica. De la misma forma se creó el Fondo Nacional de Apoyo a la ejecución de la Política de Población, el mismo que garantizaría un presupuesto adecuado para la implementación de la ley y que tenía como fuentes de financiamiento los aportes del tesoro público, aportes productos de convenios nacionales o internacionales y donaciones.

Finalmente, en el Título IV se menciona que es el Consejo Nacional de Población quien establece las inspecciones y supervisiones para una adecuada implementación de la política nacional de población.

Desde su promulgación en 1986, la Ley Nacional de Población solo ha tenido una modificatoria, que fue promulgada el 9 de setiembre del 1995, mediante Ley $\mathrm{N}^{\circ}$ 26530, la misma que modifica el artículo VI del Título Preliminar de la siguiente forma: "Artículo VI.- La Política Nacional de Población excluye el aborto como método de Planificación Familiar. En todo caso, la adopción de los métodos se basa en el libre ejercicio de la voluntad personal, sin que medien estímulos o recompensas materiales". Desde esa fecha la Ley Nacional de Población no ha vuelto a ser modificada en ninguno de sus artículos, lo que nos muestra que, a pesar de los avances, tanto demográficos como en la investigación, el tema poblacional sigue ausente del debate público y político.

Como se puede destacar, la Ley Nacional de Población significó un avance muy importante, no solo en lo referente a las políticas que en ella se plantean, sino en lo referente a la institucionalización legal que el tema poblacional empezó a tomar. Dejó de ser un tópico tratado voluntariamente por algunos gobernantes para formar parte de la preocupación y accionar del Estado, lo que se vio reflejado en los planes y programas poblacionales que se elaboraron luego de su promulgación.

Punto aparte merece una situación que ha frenado la aplicación de la Ley Nacional de Población y la posterior implementación de los Programas y Planes Nacionales de Población, hago referencia a la desactivación del Consejo
Nacional de Población. Este hecho ocurrió en 1996 con la primera disposición complementaria del Decreto Legislativo $\mathrm{N}^{\circ} 866$, de noviembre de mencionado año, en el que se crea el Ministerio de la Mujer y del Desarrollo Humano, el mismo que a partir de esa fecha asumía las funciones del Consejo. Recientemente es el Ministerio de la Mujer y Poblaciones Vulnerables quien tiene el reto y la responsabilidad de implementar el Plan Nacional de Población 2010-2014. Sin embargo, la pérdida de una institución como el Consejo Nacional de Población es un pasivo que es necesario superar para darle a la Ley Poblacional mucha mayor institucionalización ${ }^{3}$.

\section{EI Plan Nacional de Población 2010-2014}

Luego de ocho años de ausencia de un plan nacional de población, en el año 2010 se aprobó el actual Plan Nacional de Población, el mismo que fue "formulado y validado mediante consultas descentralizadas y ha contado con la participación de funcionarios públicos, expertos y líderes de la sociedad civil" (Ministerio de la Mujer y Desarrollo Social, 2010: 9).

Antes de presentar y analizar el Plan Nacional de Población 2010-2014, es necesario recordar la definición de lo que es un plan. Arlette Pichardo dice que un plan "es un documento sistemático y analítico que atiende a objetivos nacionales de desarrollo; además, resume el conjunto de decisiones en torno a los propósitos que desea lograr y a los medios para conseguirlos. Un plan es el elemento típico de la planificación estatal" (Pichardo, 1997: 37). Por lo tanto, un plan supone definir propósitos, establecer los mecanismos para el logro de los mismos, ejecutar acciones y dar a conocer los resultados logrados con dichas acciones. Un plan es tanto un proceso, ya que reune un conjunto de fases sucesivas y complementarias; y es también un sistema organizado que posibilita atender los aspectos que se ha planteado en su formulación.

Considero que la importancia de un plan para poder lograr el desarrollo adecuado de un país ha quedado suficientemente clara. El plan que en esta oportunidad analizo es el Plan Nacional de Población 2010-2014 que fue aprobado mediante Decreto Supremo No 005-2010-MINDES, del 21 de julio del año 2010 y se suma a la serie de programas y planes de desarrollo en materia poblacional elaborados por el Estado peruano ${ }^{4}$.

En la introducción del Plan Nacional de Población 2010-2014, se destaca el hecho de que éste "busca reinsertar este importante tema en las políticas, planes y programas de desarrollo social, económico y territorial, y recuperar fortaleciendo la institucionalidad encargada de su gestión a nivel nacional y regional" (Ministerio de la Mujer y Desarrollo Social, 2010: 7).

Es importante destacar que el Estado reconoce que durante muchos años (recordemos que el último Plan Nacional fue el de 1998-2002) el tema poblacional no ha contado con la preocupación de las autoridades. Por otro lado, destaca el carácter multisectorial del tema poblacional, al relacionarlo

${ }^{3}$ Es importante mencionar que también existe normatividad a nivel regional.

${ }^{4}$ El primer Programa Nacional de Población se dio en 1987, durante el primer gobierno del presidente Alan García. El segundo Programa Nacional de Población data de 1991, en los inicios del gobierno del presidente Alberto Fujimori. En 1998 se formuló el Plan Nacional de Población 1998-2002, aprobado mediante Decreto Supremo No 011-98-PROMUDEH de setiembre de 1998. 
con el desarrollo social, económico y territorial. Finalmente destaca el hecho de que ésta debe ser una preocupación del Estado en todos sus niveles de gobierno, desde el nacional hasta el distrital.

En un segundo lugar el Plan Nacional de Población aborda los principales cambios demográficos que ha experiementado la población peruana, en lo referente a los cambios en el crecimiento y distribución de la población, los cambios en la estructura etárea, los cambios en la mortalidad y la fecundidad y los cambios en la migración interna e internacional. Como se puede apreciar, la mayor parte de indicadores demográficos son tomados en cuenta en este breve diagnóstico, el mismo que concluye:

El Programa Nacional de Población 2010 - 2014 toma en cuenta este importante proceso poblacional al sugerir políticas y líneas de acción referidas a la vinculación de estos migrantes con redes de conocimiento y oportunidades de negocio en el marco del bono demográfico. Asimismo plantea una estrategia de apoyo a la migración de retorno considerando la población adulta mayor (Ministerio de la Mujer y Desarrollo Social, 2010: 26).

\section{Desafíos del Plan Nacional de Población 2010 - 2014}

En su diseño, el Plan Nacional de Población 2010-2014, plantea enfrentar algunos de los principales desafíos en materia poblacional.

El primer desafío es el del bono demográfico, situación que se da cuando la población económicamente activa (PEA) es cuantitativamente mayor a la población económicamente inactiva (PEI), lo que genera que haya un mayor potencial económico productivo. Para ello, en el Plan se propone:

[...] que se priorice la generación de inversiones productivas intensivas en trabajo, el incremento en la inversión social destinada a la capacitación laboral, políticas activas de promoción del empleo para jóvenes y el mejoramiento en la calidad de los servicios de salud ocupacional y educación técnica y superior. Es también un factor facilitador, el prever inversiones a mediano plazo para atender las demandas de la población adulta mayor de manera tal que estas 'no se coman' los ingresos y ahorros familiares (Ministerio de la Mujer y Desarrollo Social, 2010: 29).

Lamentablemente, se reconoce que la situación del bono demográfico no es ilimitada y el Perú poco a poco está llegando al año en que dicho bono demográfico llegará a su mayor nivel (año 2030). Para poder aprovechar dicha situación se requiere la generación de puestos de trabajo para la creciente población joven además del mejoramiento de los servicios de atención a la población adulta. Regiones como Lima, Tacna y Moquegua ya están llegando o han llegado a esta situación, presentándose como malos modelos de regiones que no han sabido aprovechar esta situación.

El segundo desafío que el Plan aborda es el relacionado a la explosión urbana. No es novedad decir que el proceso de urbanización del Perú se inició en la década de 1940. Según datos del XI Censo Nacional de Población, elaborado por el INEI, el $75.9 \%$ de la población nacional vive en áreas urbanas, mientras que solamente el $24.1 \%$ lo hace en el mundo rural. Las ciudades con mayor concentración poblacional urbana son Lima-Callao (con 8'472,935 habitantes), Arequipa (con 749,291 habitantes), Trujillo (con 682,834 habitantes) y Chiclayo (con 524,442 habitantes). Se destaca que: "Estas altas tasas de crecimiento urbano agravan los problemas de vivienda, saneamiento, transporte y seguridad por lo que es indispensable que sus gobiernos locales cuenten con proyecciones confiables sobre demandas futuras y el déficit en servicios básicos que deberán afrontar" (Ministerio de la Mujer y Desarrollo Social, 2010: 34).

El tercer desafío que aborda el Plan es el de la "gran dispersión de la población rural", que vendría a ser lo opuesto al desafío de la urbanización, ya que si bien es cierto que hay una mayor cantidad de población urbana, la población rural no ha dejado de crecer, solo lo ha hecho de manera más lenta. El XI Censo Nacional de Población ha identificado la existencia de 98,011 centros poblados a nivel nacional, en los cuales viven los $27^{\prime} 412,157$ de peruanos: "E1 75\% de estos centros poblados se ubican en la región de la sierra, pero aquí solamente reside el $32 \%$ de la población del país, mientras que la costa, con tan sólo el $10 \%$ de los centros poblados, aloja al $56 \%$ de la población nacional; la Selva con el $15 \%$ de los centros poblados agrupa al 13\% de la población" (Ministerio de la Mujer y Desarrollo Social, 2010: 35).

Se puede notar que los dos desafíos anteriores representan grandes retos que el Plan Nacional de Población debe afrontar, no solo por la dificultad que representan dos procesos totalmente opuestos, sino por la importancia que reviste para el desarrollo y progreso social: "Atender los problemas de la congestión urbana de un lado y el acceso a servicios básicos de una población rural muy dispersa son dos de los desafíos centrales que la política de población y de desarrollo territorial deberán atender" (Ministerio de la Mujer y Desarrollo Social, 2010: 37).

El cuarto desafío es el referido a la ampliación de la información y el acceso a métodos de planificación familiar informados, para lograr una mayor equidad en los derechos sexuales y reproductivos de toda la población. El abordaje de este desafío toma como punto central la decisión compartida, tanto de varones como de mujeres, del uso de los métodos de planificación familiar. Si bien es cierto que gracias a factores educativos la cantidad de población que hoy en día hace uso de métodos de planificación familiar ha experimentado un considerable aumento, aún cerca del $10 \%$ hacen un mal uso de alguno de los método más utilizados. ${ }^{5}$

Algunos de los factores mencionados anteriormente han generado una aumento en el número de adolescentes embarazadas, con todos los riesgos que, tanto para la madre como para los hijos, traen como consecuencia. Se concluye que:

Esta situación es reflejo de la persistencia de inequidades reproductivas en la mayor parte de departamentos del país, caracterizadas por mayores tasas de fecundidad en sectores pobres y en comunidades nativas, a lo que se añade la reproducción temprana y maternidad adolescente en

${ }^{5}$ Entre ellos podemos citar a los inyectables (17.3\%), la esterilización femenina (10.1\%), el condón (9.3\%) y la píldora (6.9\%). 
muchachas pobres y solteras, y mayores tasas de fecundidad no deseada entre mujeres en pobreza. Todo esto permite afirmar que los altos niveles de fecundidad, especialmente los que aún se registran en las mujeres adolescentes y jóvenes del país, es uno de los mecanismos que contribuyen a la reproducción intergeneracional de la pobreza. Esta situación exige una atención preferente por parte de la política y planes de población y planificación familiar, tanto a nivel nacional como regional (Ministerio de la Mujer y Desarrollo Social, 2010: 42).

Finalmente, el quinto desafío del Plan consiste en atender las necesidades y demandas que derivan del envejecimiento de la población. No se puede negar que la población peruana está envejeciendo a un paso lento, pero seguro. Según las estimaciones del INEI, "El grupo de personas de 65 y más años de edad se incrementará sostenidamente en las próximas décadas [...]. En efecto, de poco menos de 1.5 millones de adultos mayores en el 2010, pasaremos a casi 6.5 millones en 2050; 5 millones adicionales, la mayor parte de los cuales serán mujeres" (Ministerio de la Mujer y Desarrollo Social, 2010: 42). Es importante mencionar que la cantidad de población en este rango de edad (de 65 años a más) se ha incrementado como producto de la disminución de la tasa de mortalidad infantil y el incremento en la esperanza de vida, ambas generadas por los avances en medicina y tecnología de la salud, a los cuales el Perú no se ha mantenido al margen. Este desafío plantea grandes retos en materia de políticas y programas sociales para poder atender las necesidades de esta población, con la implementación de servicios de calidad acordes con las características socioeconómicas de la población de la tercera edad: "Debe tenerse en cuenta que los servicios especializados en geriatría y gerontología sólo existen en las grandes ciudades del país y no tienen la capacidad para atender estos nuevos requerimientos que plantea el proceso de envejecimiento demográfico" (Ministerio de la Mujer y Desarrollo Social, 2010: 43).

\section{Visión y Misión del Plan Nacional de Población 2010 - 2014}

El Plan Nacional de Población 2010-2014, plantea su visión:

Al 2014 el Plan Nacional de Población 2010 - 2014 contribuirá a analizar e incorporar a los planes y programas de desarrollo social y económico del país, los retos y oportunidades surgidos de los cambios demográficos a nivel nacional y regional. Asimismo, avanzará en la configuración de escenarios demográficos para el desarrollo descentralizado del país en el mediano y largo plazo (Ministerio de la Mujer y Desarrollo Social, 2010: 47).

Entendida la visión como la proyección hacia el futuro y como la construcción de una representación de la relación entre el plan y su contexto, se puede apreciar que ésta incluye diversos elementos que se han desarrollado en las partes introductorias del Plan, tales como los desafíos a enfrentar y la solución de los mismos por medio "del desarrollo descentralizado" teniendo como parámetros temporales el mediano y largo plazo. En base a esta visión se puede afirmar que el Plan Nacional de Población 2010-2014 es lo que autores como Carlos Alza (2012) clasifican como una política de Estado ya que no se circunscribe a un solo período de gobierno, sino que sobrepasa los límites temporales de una gestión presidencial ${ }^{6}$.

Por otro lado, la misión del plan plantea:

Fortalecer la capacidad del Estado, principalmente de los organismos del Gobierno Nacional y de los Gobiernos Regionales, para incorporar a los planes y programas en ejecución, las demandas y oportunidades que surgen de la transición demográfica y lograr que estos tengan mayor eficacia y eficiencia en especial en la atención a los más pobres en todas las regiones del país (Ministerio de la Mujer y Desarrollo Social, 2010: 47).

Respecto a la misión, es necesario mencionar que ésta refleja la razón de ser del Plan, dentro de su ámbito de influencia y en relación con la sociedad en su conjunto. Muestra en sí la finalidad última del Plan: "fortalecer la capacidad del Estado (en todos sus niveles) en materia poblacional", con una mayor preferencia por la población más pobre. Esta misión se caracteriza por su amplitud, ya que permite el crecimiento creativo del Plan y por su claridad de los términos en que se formula.

Como análisis final de esta parte, debo hacer notar que hay un componente fundamental, aunque con distintas denominaciones, tanto en la visión como en la misión, nos referimos al proceso de descentralización, el mismo que es tomado como objetivo final (en la visión) y como instrumento o medio (en la misión). Lo que marca el carácter descentralista intrínseco del Plan y la importancia que dicho tema tiene para una adecuada visión de país.

\section{Enfoques del Plan Nacional de Población 2010 - 2014}

Como toda política pública, el Plan se guía por una serie de enfoques que muestran el carácter multisectorial de la política en sí misma y la variedad de abordajes que puede tener, al igual que las diversas consecuencias que podría generar en la población. Estos enfoques son el de derechos, el de género, de interculturalidad, de igualdad de oportunidades y el de territorialidad.

Como se puede apreciar, la multiplicidad de enfoques que aborda el Plan nos da una idea de lo ambicioso que pretende ser al proponerse el abordaje de tantos temas. No puedo negar que este es el verdadero carácter que una política de población debe tener. Es decir, que si bien es cierto son muchos enfoques, son los necesarios para poder entender la importancia que reviste el tema poblacional, no solo como una variable más, sino como la fundamental a tomar en cuenta en todos los planes y programas de desarrollo nacional y regional. No entender esto sería negar la existencia misma del principal beneficiado y/o perjudicado con el accionar del Estado: la población misma.

6Es preciso recordar que el Plan Nacional de Población 2010-2014, se elaboró en el segundo gobierno de Alan García (2006-2011), se viene implementando en el actual gobierno de Ollanta Humala, de ahí que lo considero como una política de Estado, ya que es una propuesta que está logrando institucionalizarse en el Estado y está trascendiendo los periodos de gobierno. 


\section{Objetivos, estrategias y líneas de acción del Plan Nacional de Población 2010 - 2014}

El cuarto componente importante que se puede encontrar en el Plan es el relacionado a los objetivos, las estrategias y las líneas de acción que se han formulado para poder lograr la visión planteada y cumplir con la misión encomendada.

Hay que recordar que los objetivos son las metas que el plan desea conseguir y que representan un cambio en la situación actual en que se encuentra la situación motivo de planificación. Las estrategias representan la fase operativa del plan, ya que en ellas se explican las acciones, las formas y maneras de lograr los objetivos; es decir, definen la lógica de intervención. Finalmente, las líneas de acción constituyen el conjunto de actividades planificadas y ejecutadas para lograr, de manera eficiente y eficaz el cumplimiento de las estrategias y de los objetivos en última instancia.

Teniendo en cuenta estas consideraciones de orden conceptual, en el Plan Nacional de Población 2010-2014 se puede identificar que su objetivo general es: "Incorporar las oportunidades y retos del cambio demográfico en las políticas y estrategias de desarrollo, para contribuir a superar la pobreza y a eliminar las inequidades y desigualdades sociales, económicas y territoriales del país" (Ministerio de la Mujer y Desarrollo Social, 2010: 50). Como se puede apreciar, el objetivo general marca la utilidad que las variables demográficas tienen para las demás políticas y estrategias de desarrollo, las mismas que en perfecta armonía deben de propender al desarrollo del país. Los objetivos específicos del Plan Nacional de Población, 2010-2014, son:

1. Aprovechar la oportunidad que brinda el bono demográfico en la próxima década respondiendo al reto de priorizar la inversión en capital humano sobre todo para la población joven, en especial la afectada por la pobreza y ubicada en el medio rural y en las ciudades del interior del país.

2. Atender los retos que presentan las ciudades con gran crecimiento y congestión urbana, caracterizadas por problemas de saneamiento, seguridad, vivienda, transporte y promoción de oportunidades de trabajo y empleo, promoviendo servicios públicos de calidad y la descentralización de la inversión privada.

3. Atender las necesidades de las poblaciones para contribuir en dotarlas de servicios básicos con nuevas tecnologías en función de las potencialidades y oportunidades de cada espacio geográfico implementando las políticas para una adecuada de organización territorial.

4. Promover e impulsar el ejercicio libre, responsable e informado de los derechos de las personas, particularmente de los derechos sexuales y reproductivos.

5. Analizar y proponer políticas y programas de atención para las personas adultas mayores en especial en los ámbitos de avanzada transición demográfica para contribuir a su seguridad económica, atención de su salud y a una vida digna.

El primer objetivo aborda el desafío del bono demográfico, el mismo que debe ser afrontado de manera inexorable en la próxima década, teniendo como meta priorizar la inversión en el capital humano joven. Para ello se plantean estrategias muy claras orientadas a mejorar las condiciones de inserción laboral de la población joven, tanto en aspectos de la promoción de programas productivos, el aprovechamiento de las nuevas tecnologías de la información y el mejoramiento de la formación técnica y profesional, especialmente en las zonas más pobres.

El segundo objetivo aborda el desafío de las ciudades con gran crecimiento y congestión urbana que genera graves problemas de saneamiento, seguridad, vivienda, transporte, etc. Ante esta situación se plantean estrategias dirigidas a un diseño y planificación para un adecuado proceso de urbanización: desde la elaboración de informes de investigación demográfica, el reforzamiento de capacidades de los funcionarios de los gobiernos regionales y locales en materia de planificación y ordenamiento territorial y la conservación del medio ambiente en las grandes urbes. Todas estas estrategias tienen en común el hecho de que para poder cumplir con el objetivo, es necesario contar con información demográfica básica, elaborada por especialistas en el tema y que difundan, por medio de capacitaciones, programas de educación, publicaciones, etc., dicha información.

El tercer objetivo del Plan consiste en atender las necesidades de las poblaciones más dispersas para dotarlas de los servicios básicos, en el marco de una adecuada organización territorial. Para ello, las estrategias están orientadas a definir, ya que hasta la fecha no la hay, una política de atención a las poblaciones dispersas; la investigación en nuevas tecnologías para ampliar la cobertura de los servicios básicos en esas poblaciones y la identificación de potencialidades productivas en dichas zonas dispersas. Este objetivo representa en sí un reto mayor, ya que el tema de la dispersión poblacional no fue tomado en cuenta en anteriores planes o programas nacionales, por lo que las líneas de acción que establece, especialmente las orientadas a la investigación, son muy oportunas.

El cuarto objetivo del Plan aborda el desafío del ejercicio libre, responsable e informado de los derechos sexuales y reproductivos de las personas. Este es tal vez uno de los temas que más ha preocupado y que más se ha abordado durante toda la historia de la política de población en el Perú, temas como métodos de planificación, paternidad responsable, educación sexual y reproductiva han estado presentes en todos los planes y programas nacionales. Por estas razones, no es de extrañar que las estrategias propuestas solamente sean de fortalecimiento de los mecanismos de educación en planificación familiar, reducción del embarazo adolescente e incidencia de la Infecciones de Transmisión Sexual (ITS) y el VIH/SIDA.

Finalmente, el quinto objetivo del Plan propone el diseño de políticas y programas de atención para las personas adultas mayores, en especial en las zonas donde la transición demográfica está avanzada. Al ser este un tema relativamente novedoso en nuestro país, no es de extrañar que la primera estrategia tenga que ver con la visión más cortoplacista del problema; es decir, con la orientación de los servicios públicos hacia las necesidades especiales de esta población. La segunda estrategia, tiene un corte de mediano y largo plazo, ya que se propone la elaboración de estudios para un programa de apoyo a las personas adultas mayores en situación de abandono, especialmente en las zonas rurales.

Como se puede apreciar, los cinco objetivos específicos del Plan están orientados a abordar los desafíos planteados líneas arriba y que representan temas de preocupación 
nacional. A su vez estos objetivos tienen relación con las líneas directrices establecidas en el Decreto legislativo $\mathrm{N}^{\circ}$ 346, Ley de la Política Nacional de Población.

\section{Mecanismos institucionales para la gestión del Plan Nacional de Población 2010-2014}

El Plan Nacional de Población 2010-2014 concluye con un aspecto muy importante, me refiero al establecimiento de los mecanismos institucionales indispensables para la gestión del mismo. Es muy importante recordar que "la implementación depende en gran medida de la calidad de las instituciones que intervienen" (Ortegón, 2008: 49). Dichas instituciones definen los roles de los actores, sus responsabilidades e interacciones, por lo que la implementación de las políticas públicas depende en gran medida de la calidad de sus instituciones y de la interacción e intereses de los actores que en ella intervienen.

La primera estrategia que establece el Plan en materia institucional es la de "fortalecer la rectoría del MIMDES y las competencias de las instituciones públicas, nacionales y regionales, para la implementación, seguimiento y evaluación del Plan nacional de Población 2010-2014" (Ministerio de la Mujer y Desarrollo Social, 2010: 75). Dentro de esta estrategia, las líneas de acciones más importantes están referidas al proceso de descentralziación de la institucionalidad del MIMDES en materia poblacional, para ello se propone crear instancias orgánicas regionales, brindar apoyo técnico a los gobiernos regionales y formular los programas regionales de población. Todas estas acciones no se podrían realizar sin un presupuesto determinado, por lo que es importante destacar el hecho de que se compromete una partida presupuestal en materia de población en los presupuestos a nivel regional y local.

La segunda y última estrategia institucional es la de "promover el conocimiento y el consenso sobre los desafíos que la dinámica demográfica supone para el desarrollo del país" (Ministerio de la Mujer y Desarrollo Social, 2010: 76). Esta estrategia se encuentra íntimamemente relacionada con los temas de elaboración, capacitación y difusión de material educativo en población y la consecuente implemantación de campañas de comunicación. El objetivo de esta estrategia es claro: lograr que no solo el Estado asuma un compromiso respecto al tema poblacional, sino que la propia población sea conciente de la responsabilidad que tiene frente a este tema.

Para finalizar el análisis del Plan Nacional de Población 2010-2014, es importante mencionar que para poder cumplir con sus objetivos, el decreto supremo $\mathrm{N}^{\circ}$ 005-2010-MIMDES, establece la constitución de la Comisión Multisectorial Permanente que estará adscrita al Ministerio de la Mujer y Desarrollo Social (actual MINVP) e integrada por: un representante del Ministerio de la Mujer y Desarrollo Social (quien presidirá la Comisión), un representante de la Presidencia del Consejo de Ministros, un representante del Ministerio de Economía y Finanzas, un representante del Ministerio de Salud, un representante del Ministerio de Educación, un representante del Ministerio de Trabajo y Promoción del Empleo, un representante del Ministerio del Ambiente, un representante del Ministerio de Vivienda, Construcción y Saneamiento, un representante del Ministerio de Transportes y Comunicaciones, un representante del Centro Nacional de Planeamiento Estratégico y un representante del Instituto Nacional de Estadística e Informática. Esta comisión estará encargada de la implementación, seguimiento y evaluación del Plan.

Uno de los objetivos principales que se deben de cumplir como parte del proceso de implementación del Plan Nacional de Población es que cada región elabore su programa regional de población. Sobre el caso particular de la implementación de la política de población en la región Arequipa, se puede consultar a Mario Berrios (2015).

\section{A modo de conclusión}

Desde la década de los años 1970, el Perú experimentó una etapa de profundización de grandes cambios y transformaciones sociales, económicas, políticas y demográficas. Dichos cambios se agudizaron gracias al proceso de migración del campo a la ciudad, lo que revirtió el porcentaje de población rural y urbana. Aunado a ello, los avances científicos en medicina y la masificación de la educación, permitieron la reducción de las tasas de mortalidad, las tasas de mortalidad infantil y las tasas de fecundidad. Sin embargo, pese a estos importantes cambios en la dinámica de la población, el componente poblacional fue considerado muy poco al momento de la elaboración de las políticas públicas.

Es a partir de la intervención del Perú en la Conferencia Mundial de Población de 1974, organizada por el Fondo de Población de las Naciones Unidas, que se inicia una etapa de preocupación por el componente poblacional y las consecuencias socioeconómicas que éste tiene sobre el desarrollo nacional. En ese sentido, destaca el accionar del Estado con la creación de organismos públicos y la elaboración de un cuerpo legal de normas que fueron las bases para lo que hoy conocemos como la política pública de población.

A la fecha se cuenta con un plan nacional de población desactualizado, que no se ha implementado adecuadamente en todas las regiones del país, ello principalmente a la poca voluntad política de las autoridades y a débil institucionalidad del gobierno en materia poblacional.

\section{REFERENCIAS BIBLIOGRÁFICAS}

1. Alza, Carlos (2012), Maestría en Ciencia Política y Gobierno. Curso 1: Diseño y Evaluación de Políticas Públicas. Lima: PUCP.

2. Berrios, Mario (2015), Del dicho al hecho. La brecha de implementación en la política pública de población. Arequipa: UCSM-Texao.

3. Cueto, Marcos (2006), "La vocación por volver a empezar: las políticas de población en el Perú". Revista Peruana Médica. Lima, volumen 23, número 2, pp. 123-131.

4. Donayre, José y otros (2012), Políticas y programas de población en el Perú: Del debate a la acción. Lima: Universidad Peruana Cayetano Heredia.

5. Ministerio de la Mujer y Desarrollo Social (2010), Plan Nacional de Población 2010-2014. Lima: s/e.

6. Ministerio de la Mujer y Desarrollo Social (2011), Guía metodológica para la formulación de políticas y programas de población en el ámbito de los Gobiernos regionales. Lima: s/e. 
7. Ministerio de Salud (1976), Decreto Supremo 0062576-MS. 31 de agosto.

8. Necochea, Raúl (2016), La planificación familiar en el Perú del siglo XX. Lima: IEP, UNFPA.

9. Ortegón, Edgar (2008), Guía sobre diseño y gestión de la Política Pública. Bogotá: Instituto de Estudios Latinoamericanos.

10. Pichardo, Arlette (1997), Planificación y programación social. Bases para el diagnóstico y la formulación de Programas y Proyectos Sociales. Buenos Aires: LumenHumanitas. 